\title{
Pseudomonas aeruginosa Preparation
}

National Cancer Institute

\section{Source}

National Cancer Institute. Pseudomonas aeruginosa Preparation. NCI Thesaurus. Code C97662.

A preparation containing the inactivated Pseudomonas aeruginosa with potential immunomodulating activity. Upon inoculation, Pseudomonas aeruginosa preparation may stimulate the immune system, increasing macrophage and natural killer cell activity; it may be used thereby in cancer adjuvant treatments, and it may reduce the incidence of infection. 